\title{
Spectrum Sharing Optimization in Cellular Networks under Target Performance and Budget Restriction
}

\author{
Md Asaduzzaman, Raouf Abozariba* and Mohammad N. Patwary \\ $\{$ md.asaduzzaman $\},\{$ r.abozariba $\},\{$ m.n.patwary\}@staffs.ac.uk \\ Faculty of Computing, Engineering and Sciences (FCES), \\ Staffordshire University, Stoke-on-Trent, Staffordshire ST4 2DE, United Kingdom
}

\begin{abstract}
Dynamic Spectrum Sharing (DSS) aims to provide opportunistic access to under-utilised spectrum in cellular networks for secondary network operators. In this paper we propose an algorithm using stochastic and optimisation models to borrow spectrum bandwidths under the assumption that more resources exist for secondary access than the secondary network demand by considering a merchant mode. The main aim of the paper is to address the problem of spectrum borrowing in DSS environments, where a secondary network operator aims to borrow the required spectrum from multiple primary network operators to achieve a maximum profit under specific grade of service $(\mathrm{GoS})$ and budget restriction. We assume that the primary network operators offer spectrum access opportunities with variable number of channels (contiguous and/or non-contiguous) at variable prices. Results obtained are then compared with results derived from an algorithm in which spectrum borrowing are random. Comparisons showed that the gain in the results obtained from our proposed stochastic-optimisation framework is significantly higher than random counterpart.
\end{abstract}

\section{INTRODUCTION}

Cellular data networks have recently witnessed rapid growth, especially due to the emergence of smartphones which led to an ever-increasing demand for wireless services in certain licensed and unlicensed spectrum bands [1]. To meet the growing traffic demand, significant technology improvements to enhance spectral efficiency is required. Static partitioning of spectrum, for example, has significant operational implications (e.g., pseudo scarcity of the available radio spectrum) which have been identified by extensive spectrum utilisation measurements in Europe and the USA [2]. These measurements show that a large part of the radio spectrum which is allocated to cellular use are quite well utilised but the utilisation varies dramatically over time and space. Such variation of spectrum utilisation causes the so-called spectrum holes.

The current static spectrum management must give way to a new approach that breaks down artificial spectrum access barriers and enables networks and their subscribers to dynamically access the spectrum [3], [4], [5]. As a response, for example, in the UK, there are plans for spectrum liberalisation between operators with different spectrum holdings [6]. Liberalisation of spectrum in the hands of the incumbent holders, and mandatory spectrum release, means some liberalised spec- trum ends up in the hands of a third party for secondary use. It is also possible that spectrum will be redistributed not only because of such a mandate, but also as a result of secondary market trading [7], [8], [9], [1]. Such secondary trading of spectrum will enhance the overall spectrum utilisation. As a result, service providers would be allowed to release their under-utilised commodities to the potential buyers [10], [11].

With the large number of service providers in the mobile cellular networks industry, each with their own policy and strategy, a variety of spectrum opportunities could be available for secondary use. To this end, in order to distinguish between options of different bandwidth releases, incumbent holders of spectrum licenses may broadcast information in relation to these available bandwidths for possible leasing to secondary operators [12]. Part of the information broadcasted by the incumbent spectrum holders are in the form of available spectrum for access size, location boundaries, maximum transmit power, duration of the lease and admission cost [13].

Operators aim to provide a stable GoS to their respective users, even if it would incur additional cost. However, in a scenario where the number of wireless cellular operators is high, making dynamic, on-demand and correct choice of acquiring additional bandwidths can result in profit maximisation for a given operator, especially when operators provide service for large territories.

In general, this paper focuses on the predicted future demand for mobile data services which may be provided using liberalised spectrum and spectrum trading [14], [15], [16]. Given a market scenario and other inputs, we propose a technical algorithm with the aim to optimise the profit of secondary operators, which are likely to be achieved by an operator with a given number of cells. We also evaluate spectrum borrowing scenarios by incorporating a large number of cells. Major contributions of this paper are as follows:

- a novel purchase approach for dynamic spectrum sharing (DSS) network is proposed in the presence of multiple primary service operators. We introduce an optimisation problem in merchant mode DSS and we solve the problem by using stochastic modelling and mathematical programming, 
- the robustness of the proposed algorithm is investigated in the presence of large number of cells and various types of spectrum bands and the proposed algorithms compared with random borrowing algorithm. Comparisons showed a substantial gain over the random borrowing algorithms and

- incorporation of the proposed model will complement the well studied auction mode spectrum trading schemes, while preparing automation of licensing by frequency regulatory bodies.

\section{RELATED WORK}

In the literature, a great number of studies has appeared in recent years on the design of dynamic spectrum sharing within cellular networks. Interests in this context include secondary leasing and pricing strategies among incumbent spectrum license holders, secondary operators and secondary users. These prior studies mainly focused on approaches using auction mode and game theory to implement the spectrum pricing and allocation schemes by taking into account the variation of the networks demands and constraints such as power, price and interference [17], [18], [19], [20], [21], [22].

In [23], the authors proposes a multiple-dimension auctioning mechanism through a broker to facilitate an efficient secondary spectrum market. In [24] a knapsack based auction model that dynamically allocates spectrum to the wireless service providers (WSPs) such that revenue and spectrum usage are maximised. A dynamic pricing strategy for the service providers is also proposed. Auction schemes where a central clearing authority auctions spectrum to bidders, while explicitly accounting for communication constraints is proposed in [13]. The used techniques are related to the posterior matching scheme, which is used in systems with channel output feedback. While in [25], spectrum auctions in a dynamic setting where secondary users can change their valuations based on their experiences with the channel quality was studied. Price-based DSS has also been investigated from the business perspective [26], [7]. For example, In [27] An extensive business portfolio for heterogeneous networks is presented to analyse the benefits due to multi-operator cooperation for spectrum sharing. High resolution pricing models are developed to dynamically facilitate price adaptation to the system State. In [28], a quality-aware dynamic pricing algorithm (QADP) which maximises the overall network revenue while maintaining the stability of the network was studied.

The vast majority of the aforementioned studies consider competitive market scenarios and therefore auction and game theory have been discussed to develop DSS strategies. By using the same assumption, pricing in the context of DSS has mainly been considered from the spectrum owners perspective to maximise their revenues [26], [21], [29]. However, when the number of available bandwidths from multiple license owners is higher than SNO's demand, then auction mode is not always the best strategy. This is because the number of bidders might be too small and the best selling price can not be achieved for the license owners by using auction mode. A more realistic and pragmatic model in this case is a merchant mode, which to the best of the authors' knowledge, has not been investigated in the context of DSS. Moreover, spectrum borrowing when considering budget restrictions has not been addressed. Also, there is currently no published work, which attempted to study the profit maximisation in merchant mode with target performance. Thus, the problem that we formulate and solve substantially differ from those available in the literature.

The paper is organised as follows: the proposed dynamic spectrum management model is described in Section III. Section IV addresses the problem of spectrum allocation in homogeneous cellular networks and describes our mathematical programming formulations to the problem. In Section V, we present our findings. Finally, Section VI summarises our conclusions.

\section{DYNAMIC SPECTRUM MANAGEMENT MODEL}

\section{A. System overview}

We consider a cellular network consists of one secondary network operator (SNO) and $\mathcal{N}$, with size $|\mathcal{N}|=N$, denote the set of primary network operators (PNOs) serving a region $\mathcal{R}$, see Figure 1 . Let $\mathcal{L}$, with size $|\mathcal{L}|=L$, be the set of cells in the region. The operators consist of base stations and Radio Network Controller (RNC). The main functions of the RNC are to monitor base stations and to assign resources for the air interface. Due to spectrum liberalisation, the PNOs $|\mathcal{N}|$ will have the freedom to lease their spectrum bandwidths to the SNO. Leasing spectrum bandwidths to SNO would mean that the secondary system will have to pay a certain compensation to the primary system for using the spectrum bandwidths, and naturally the amount of compensation can be expected to be proportional to the amount of allowed spectrum leasing by the primary system. We assume the compensation paid to the PNO is in form of monetary value. The PNOs broadcast specific information about their available bands for leasing and admission cost (per unit bandwidth) at each cell $i \in \mathcal{L}$ on fixed identical intervals (e.g., every 2 hours). More specifically, the PNOs only grants a time-bound lease to the SNOs and the lease conditions may specify additional parameters, such as the extent of spatial region for spectrum use and maximum power. The compliant use of leased spectrum requires the SNO to return the spectrum to the PNO at the end of the lease interval.

\section{B. Spectrum trading}

We consider a spectrum market based on merchant mode where PNOs independently determine the size of the available spectrum for lease along with the associated monetary value. The price of the available primary spectrum can be determined by estimating the utilisation and demand in time and space [30]. The available number of channels and the associated prices are then advertised according to a take-it or leave-it policy. No negotiation or bidding is conducted among network operators. Merchant mode is appropriate when the demand from the SNO is less than the available spectrum [4]. For analytical purposes we have evaluated the proposed algorithms 


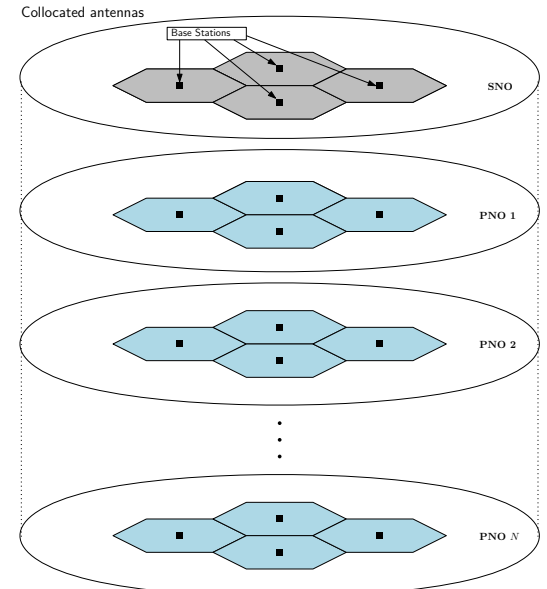

Fig. 1: Service operators sharing network

even for the case where the demand is higher than the available spectrum, but only in a fraction of the controlled cells.

\section{PROBLEM FORMULATION}

Considering the system model described in the previous section, the problem now becomes how the SNO acquires additional spectrum from PNOs. The spectrum borrowing for an SNO can be performed by considering the following objective:

- borrow the required spectrum from multiple PNOs to achieve a specific grade of service for SNO under maximum and budget restrictions.

In principle, the SNO's objective is to maximise revenue as well as to maximise utility to the end users. We formulate the problem as a finite horizon nonlinear stochastic program whose computation time is polynomial in the input size.

\section{A. Modelling assumptions}

We identify the part of network information which is assumed to be known to the SNO:

- arrival rate of the SNO at $i$ th cell for $j$ th type of spectrum band $\lambda_{i_{j}}, \forall i, j$,

- service rate of the SNO at $i$ th cell for $j$ th type of spectrum band $\mu_{i_{j}}, \forall i, j$,

- available bandwidth of the SNO at $i$ th cell for $j$ th type of spectrum band $w_{i_{j}}, \forall i, j$,

- borrowing cost of the SNO for unit bandwidth from the PNOs at $i$ th cell for $j$ th type of spectrum band $c_{i_{j} k}, \forall i, j, k$ (which are assumed to be announced periodically by the PNOs),

- allocated budget for borrowing bandwidths to the SNO at $i$ th cell for $j$ th type of spectrum band from the PNOs $b_{i_{j}}, \forall i, j$,

- available bandwidth of the $k$ th PNO at $i$ th cell for $j$ th type of spectrum band $a_{i_{j} k}, \forall i, j, k$, (which are assumed to be announced periodically by the PNOs), and

- expected profit of the SNO at $i$ th cell for $j$ th type of spectrum band for borrowing unit bandwidth from $k$ th PNO $\alpha_{i_{j} k}, \forall i, j, k$.
Time is divided into equal-length slots $\mathcal{T}=\{0,1,2, \ldots\}$. At each time slot $t \in \mathcal{T}$ the process of channel borrowing is repeated. We use the time indicator $(t)$ to emphasise the vectors dependancy in time. Trading of bandwidth is done between primary and secondary providers separately in each of successive time windows of a particular duration. Henceforth, we focus on the the process of channel borrowing and optimisation in a single window.

\section{B. Notations used:}

Let us define the following quantities which are used later in mathematical programming problems (Problem 1 and Problem 2):

$c_{i_{j} k}(t):=$ cost of unit bandwidth to be borrowed from $k$ th PNO for $j$ type resource at $i$ th cell during time interval $t$, where $c_{i_{j} k}(t) \in \mathbb{R}_{\geq 0}^{L \times N_{i_{j}}}$.

$x_{i_{j} k}(t):=$ unit of spectrum bandwidths (or sub-bands) to be borrowed from $k$ th PNO for $j$ type resource at $i$ th cell during time interval $t$, where $x_{i_{j} k}(t) \in \mathbb{R}_{\geq 0} \times N_{i_{j}}$.

$\theta_{i_{j} k}(t):=$ PNOs intrinsic quality (e.g., the extent of the coverage area and/or maximum allowable transmit power), where $\left\{\theta_{i_{j} 1}, \theta_{i_{j} 2}, \ldots, \theta_{i_{j} k}, \ldots, \theta_{L \times N}\right\}$.

$p_{i_{j}}(t):=$ target blocking probability for $j$ type of spectrum band at $i$ th cell during time interval $t$ for the secondary network operator.

$a_{i_{j} k}(t):=$ unit bandwidth available from $k$ th PNO to be leased to SNO for $j$ th type of spectrum band at the $i$ th cell during time interval $t$, where $a_{i_{j} k}(t) \in \mathbb{R}_{\geq 0}^{L \times N_{i_{j}}}$.

$r_{i_{j}}(t):=$ unit bandwidth required to satisfy the target blocking probability $p_{i_{j}}(t)$ for the SNO's for $j$ th type of spectrum band at $i$ th cell during time interval $t$, where $r_{i_{j}}(t) \in \mathbb{R}_{\geq 0}^{L}$.

$\alpha_{i_{j} k}(t):=$ the expected profit for borrowing unit bandwidth from $k$ th PNO for $j$ th type of spectrum band at $i$ th cell during time interval $t$, where $\alpha_{i_{j} k}(t) \in \mathbb{R}^{L \times N_{i_{j}}}$.

\section{Profit maximisation under restricted budget}

In this section, we formulate the spectrum allocation problem that illustrates how much spectrum bandwidths to be borrowed from each PNO to keep the blocking probability in a specific level. Given a set of possible available spectrum resources $\left\{a_{i_{j} k}(t)\right\}$, their associated prices $\left\{c_{i_{j} k}(t)\right\}$ and expected profit $\left\{\alpha_{i_{j} k}(t)\right\}$, the problem is to find the feasible set of spectrum bandwidths $\left\{x_{i_{j} k}(t)\right\}$ by maximising the total profit of the SNO, under allocated budget. Resource acquisition in this case is obtained by solving the following optimisation problem:

\section{Problem:}

$$
\operatorname{maximise}\left[\sum_{i=1}^{L} \sum_{j=1}^{M} \sum_{k=1}^{N_{j}} \alpha_{i_{j} k}(t) \cdot x_{i_{j} k}(t)\right]
$$


subject to

$$
\begin{aligned}
\underset{x_{i_{j}} \forall i, j, k}{\arg \min } \operatorname{Pr}\left(\lambda(t), \mu(t), r_{i_{j} k}(t)+w_{i_{j}}\right) & \leq p_{i_{j}}(t), \quad \forall i_{j}, k(2) \\
x_{i_{j} k}(t) & \leq a_{i_{j} k}(t), \quad \forall i_{j}, k(3) \\
\sum_{k=1}^{N_{i_{j}}} x_{i_{j} k}(t) & \leq r_{i_{j}}(t), \quad \forall i_{j}, k \quad(4) \\
\sum_{k=1}^{N_{j}} c_{i_{j} k}(t) \cdot x_{i_{j} k}(t) & \leq b_{i_{j}} \quad \forall i_{j}, k,
\end{aligned}
$$

where $\alpha_{i_{j} k}(t)$ consists of two parts: the expected revenue $v_{i_{j}}(t)$ and cost $c_{i_{j} k}(t)$, which can be obtained as

$$
\alpha_{i_{j} k}(t)=v_{i_{j} k}(t)-c_{i_{j} k}(t)
$$

where

$$
v_{i_{j} k}(t)=f\left(x_{i_{j} k}(t), \theta_{i_{j} k}(t)\right)
$$

Here, we consider the intrinsic quality per unit bandwidth $\left(\theta_{i_{j} k}(t)\right)$ for each PNO, which can vary, i.e., $\theta_{i_{j} k}(t) \lesseqgtr \theta_{i_{j} l}(t)$, $\forall i_{j}$ and $\forall k, l$ with $k \neq l$. In this problem formulation, the parameter $\theta_{i_{j} k}(t)$ influences the optimal spectrum borrowing decisions.

Typically, the revenue $v_{i_{j} k}(t)$ earned through the sale of the borrowed bandwidth must exceed the borrowing cost, i.e., $v_{i_{j} k}(t)>c_{i_{j} k}(t)$ due to the assumption that profit of the PNO for borrowing a unit bandwidth is always positive $\left(\alpha_{i_{j} k}(t)\right)$. The inequality constraint in equation (5) implies that the SNO maximises its profit by taking into account the limitations imposed by cost of the utility and the maximum allowable expenditure which the SNO can spend for borrowing spectrum demand in each cell. Next, we solve the above non-linear optimisation problem in two phases:

\section{- Phase 1: Stochastic modelling}

The SNO set the target blocking probability for each cell (e.g., $p_{i_{j}}=0.01, \forall i_{j}$ ). Then it calculates the bandwidth $r_{i_{j}}(t)$ required to achieve the target blocking probability $p_{i_{j}}(t)$ for each cell $i$. Next, the SNO finds the amount of bandwidth required to borrow from primary networks. Blocking probability at the $i$ th cell of SNO can be defined as

$$
P_{(b)}(t)=\frac{1}{v !}\left(\frac{\lambda(t)}{\mu(t)}\right)^{v}\left[\sum_{n=0}^{v} \frac{1}{n !}\left(\frac{\lambda(t)}{\mu(t)}\right)^{n}\right]^{-1} .
$$

Now with the initial fixed bandwidth $w_{i_{j}}$, we first cal-

culate the total required bandwidth $\tau_{i_{j}}(t)$ to achieve the target blocking probability for the $i$ th cell of the SNO

$$
\tau_{i_{j}}(t)=f^{-1}\left(\operatorname{Pr}\left(\lambda_{i_{j}}(t), \mu_{i_{j}}(t), w_{i_{j}}\right)\right) .
$$

where $f^{-1}(\cdot)$ is the inverse function of $P_{(b)}(t)$ (equation 8 ) used to derive the required capacity over the existing capacity.
Subtracting the fixed bandwidth $w_{i_{j}}$ from the total required $\tau_{i_{j}}(t)$, we obtain the required bandwidth $r_{i_{j}}(t)$ at the $i$ th cell of the SNO during time interval $t$

$$
r_{i_{j}}(t)=\tau_{i_{j}}(t)-w_{i_{j}} .
$$

Now the problem is to find the feasible set of bandwidth $x_{i_{j} k}(t)$ from the PNOs which minimises the borrowing cost. This is realised in the next mathematical programming phase. The spectrum demand is adjusted dynamically based on the network information provided by the expected cell demand, service rate and existing spectrum bandwidth.

\section{- Phase 2: Mathematical programming}

In this phase, we set up the vectors $\left\{c_{i_{j} k}(t)\right\},\left\{a_{i_{j} k}(t)\right\}$ and $\left\{\alpha_{i_{j} k}(t)\right\}$. The borrowing decisions of the SNO are made subject to achieving the maximum profit for each acquisition from the PNOs. In this formulation, the borrowing capacity of the SNO is restricted to budget allocation $b_{i_{j}}$. If the acquired resources $a_{i_{j} k}(t)$ are insufficient to reach the target blocking probability $p_{i_{j}}(t)$ (i.e., $r_{i_{j} k}(t)-a_{i_{j} k}(t)>0$ ), then the SNO borrows from the remaining bandwidths from the set $\left\{a_{i_{j} 1}(t), a_{i_{j} 2}(t), \ldots, a_{i_{j} N}(t)\right\} \not \nexists a_{i_{j} k}(t)$ for which the cost is minimum. If the required blocking probability $p_{i_{j}}(t)$ is reached, then the SNO stops acquiring new spectrum bandwidths until the next time interval $(t+1)$.

Next, we list the detailed procedure in Algorithm 1.

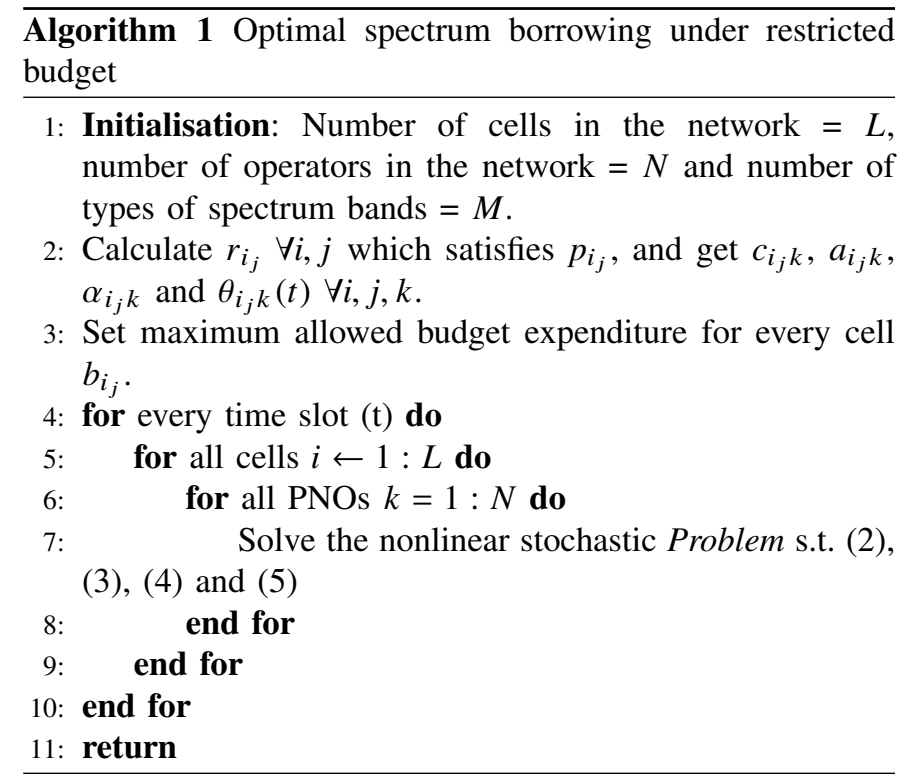

\section{Spectrum allocation using random algorithm under budget constraint}

In this subsection, we solve the problem of spectrum allocation under budget constraint by a random bandwidth selection algorithm (Algorithm 2). The algorithm performs all the steps as in Algorithm 1. However, Algorithm 2 does not perform spectrum selection according to the highest possible profit combination from the set $\left\{a_{i_{j} k}\right\}$, rather runs on randomly 
selected combination from the set $\left\{a_{i_{j} k}\right\}$ to satisfy the spectrum demand $r_{i_{j}}$. To satisfy the constraints in equation (3), (4) and (5) we use

$$
x_{i_{j} k}(t)= \begin{cases}a_{i_{j} k}(t), & r_{i_{j}}(t) \geq a_{i_{j} k}(t), b_{i_{j}} \geq c_{i_{j} k}, \\ r_{i_{j}}(t), & r_{i_{j}}(t)<a_{i_{j} k}(t), b_{i_{j}} \geq c_{i_{j} k}, \\ 0, & b_{i_{j}}<c_{i_{j} k} \text { or } r_{i_{j}}(t)=0 .\end{cases}
$$

Note that when $\sum a_{i_{j} k} \leq r_{i_{j}}$ the feasible set $\left\{x_{i_{j} k}\right\}$ is equal for both formulations. We also note that when

$$
\sum_{k=1}^{N_{i_{j}}} a_{i_{j} k}(t)>r_{i_{j}}(t),
$$

the optimal and random algorithm may achieve the same outcome in terms of total borrowing cost, however, this is a result of randomness in the selection process with probability

$P$ (selecting optimal bandwidths)

$$
= \begin{cases}\frac{1}{N} \quad & a_{i_{j} k} \geq r_{i_{j}}, \forall i_{j} \\ \frac{1}{\mid\left\{\bar{a}_{i_{j} . .} \mid\right.} & \sum_{m}\left\{\bar{a}_{i_{j} l m}, \forall l, m\right\} \geq r_{i_{j}}, \forall i_{j} \\ 1 & \sum_{k=1}^{N_{i_{j}}} a_{i_{j} k} \leq r_{i_{j}}, \forall i_{j}\end{cases}
$$

where $\left\{\bar{a}_{i_{j} l m}, \forall l, m\right\} \subset\left\{a_{i_{j} k}, \forall i_{j}, k\right\}$, and $\left|\left\{\bar{a}_{i_{j} . .}\right\}\right|$ is the number of subsets in the set $\left\{\bar{a}_{i_{j} . .}\right\}$ which satisfy the bandwidth requirement for the $i$ th cell with $j$ th type of spectrum band.

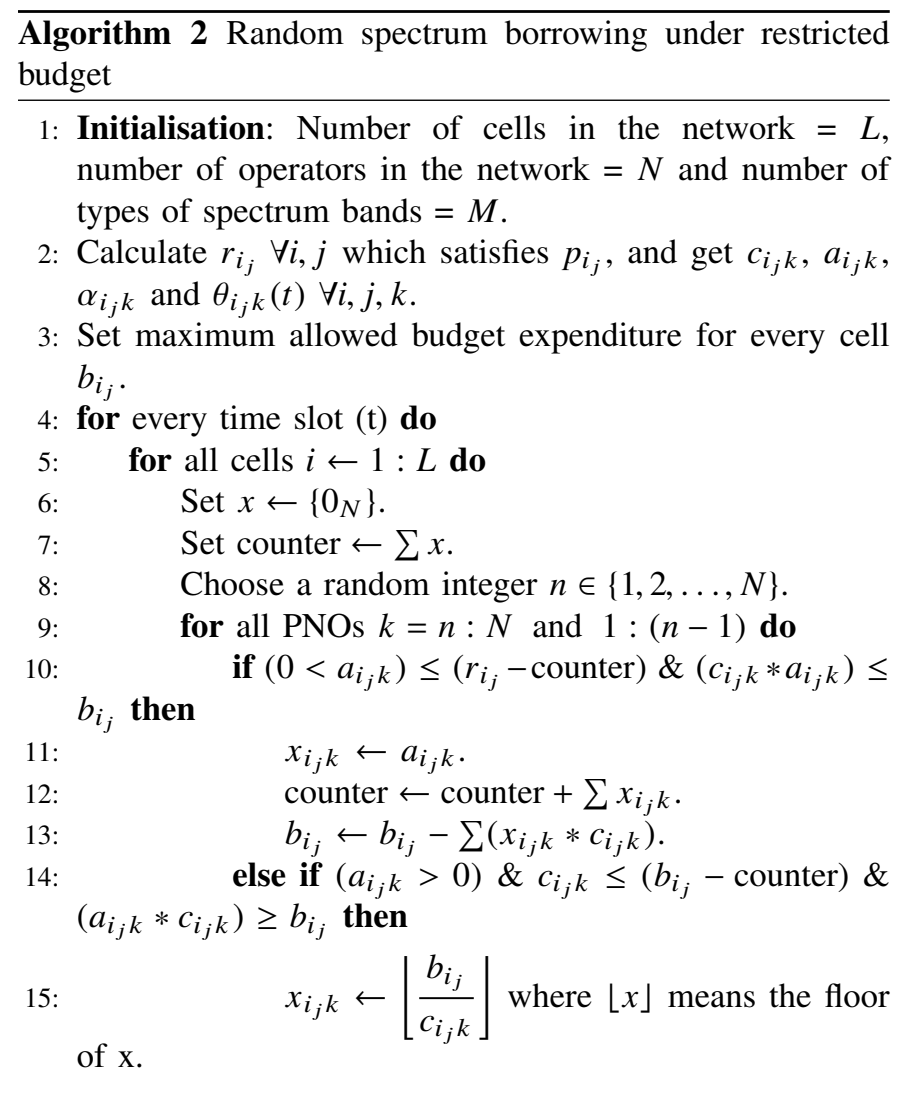

$\overline{\text { Algorithm } 2 \text { Random spectrum borrowing under restricted }}$ budget (continued)

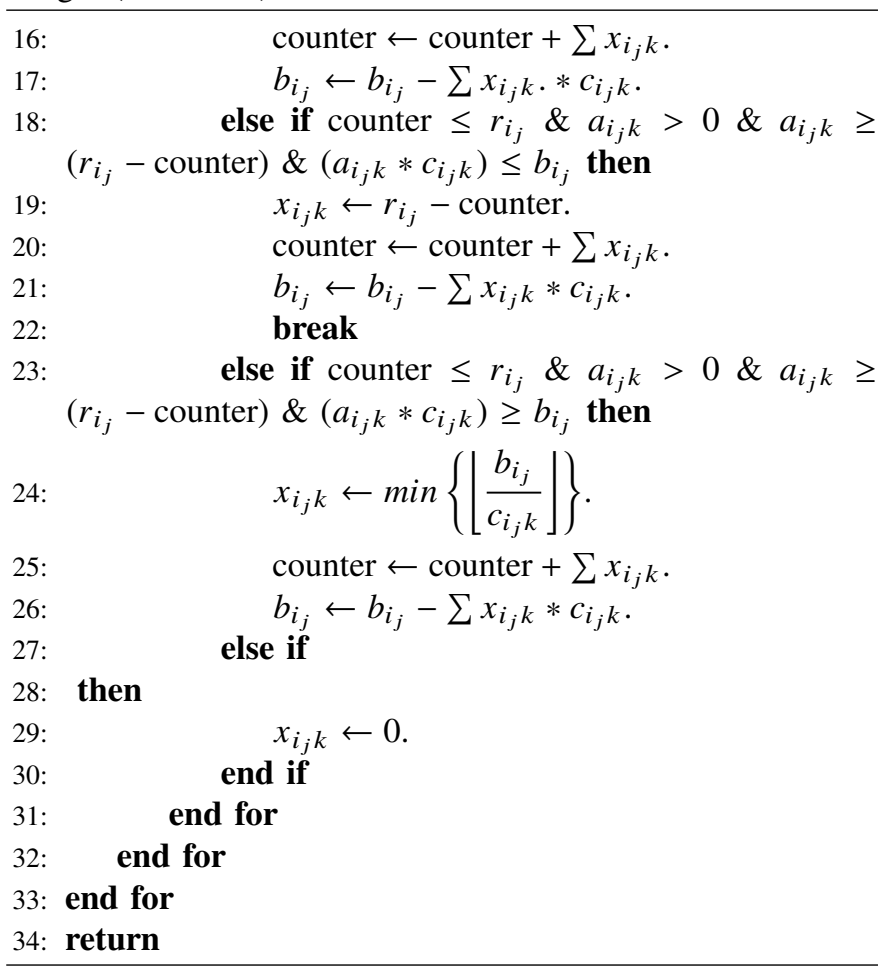

\section{RESULTS AND ANALYSIS}

In this section, we show the analysis of optimal borrowing solutions by Algorithm 1 which describes the profit maximisation with restricted budget scenarios. To explore the advantages of the proposed formulation, we compare the results from Algorithm 1 with a random spectrum selection formulation by Algorithm 2. We simulate the functionalities of the network management which are necessary to generate the optimal solution and to compare with the random spectrum selection algorithms. We consider one SNO and four PNOs $(N=4)$ to simulate the dynamics of the merchant mode resource sharing mechanism. Some parameters are determined randomly by the algorithms with specific distribution (e.g., $\lambda_{i}$, $\mu_{i}, w_{i}$ ) and we control other parameters (e.g., $L, p_{i_{j}}$ ). The algorithms are tested for different scenarios subject to those network parameters.

\section{A. Expected profit under budget constraints analysis}

The objective of the SNO can be described both from economic and system performance perspective. Firstly, the SNO aims to lower the blocking probability for its subscribers. Secondly, the SNO attempts to maximise its profit by leasing additional spectrum from the PNOs in terms of cost and intrinsic quality. However, since network operators often operate with limited budget e.g., SNO can only spend $b_{i_{j}}(t)$ amount of resources/money at a cell $i$ and time interval $t$. This could be imposed by the government and regulatory bodies to keep the fairness of spectrum leasing among network operators.

To demonstrate the gain by the optimal algorithm, detailed investigation has been made and the results are compared 
with the random allocation algorithm (see Figure 2a). The optimal algorithm shows a substantial gain is achievable in comparison to using the random allocation approach. However, both algorithms provide acceptable efficiency in terms of GoS. We also notice that as the number of cells increase the profit of the SNO gets larger, see Figure $2 b$.

We also study how the optimal allocation based on profit maximisation affects the amount of acquired bandwidths. With number of cells between $1-100$, we compare the two algorithms presented in Section IV, see Figure 3. We find that, the optimal algorithm can achieve higher number of aggregated channels due to the higher efficiency in spectrum borrowing under the restricted budget.

\section{B. Expected profit under budget constraints with multiple types of band}

In the above analysis, we considered only one type of band $(M=1)$, which is provided to users at all cells (e.g., 900 $\mathrm{MHz}$ ). In a more general model, different types of bands (e.g., $900 \mathrm{MHz}, 2.3-2.4 \mathrm{GHz}$ and 2.40-2.4835 GHz) can be operated by one network operators. Different bands provide different quality in the mobile broadband services [1]. The measures of quality include data rate and coverage. Therefore, they cannot be treated equally. In the proposed algorithm, we added a functionality to allow the management of spectrum trading to be more effective by assigning each cell with a particular band type. In order to quantify the impact of the proposed algorithms we simulated a network which could support three different bands, $(M=3)$. We also tested the algorithms with two different budgets. In the simulation of 10 cells and allocated budget of 50 and 500 for each cell, we observed a markedly increased profit in both cases, see Figure 4. We can also see from the figures (top and bottom figures) that in all types of bands, the optimal algorithm outperforms its random counterpart.

\section{CONCLUSION}

We have presented a finite horizon nonlinear optimisation stochastic program to solve an optimisation problem for dynamic spectrum sharing. The problem is investigated by considering a comprehensive process of delivering the SNO's bandwidth demand and the solution algorithm ensured that maximum profit under budget restrictions are achieved. The SNO aims to achieve a target performance by borrowing spectrum from other network operators (PNOs) on a temporal and spatial basis. Results obtained from the proposed model is then compared with results derived from an algorithm in which spectrum borrowing is random. Detailed comparisons are presented and they showed that the gain in the results obtained from our proposed stochastic-optimisation framework is markedly higher than random borrowing algorithm. Our proposed approaches facilitate a dynamic purchasing (also called automation of licensing) scheme for such a complex problem, which provides incentives to the network operators wishing to adopt dynamic spectrum sharing as well as substantial benefits for efficient use of spectrum. This is a natural implementation of the stochastic and optimisation framework which to the best of the authors' knowledge has not been addressed in previous works in this context. The proposed algorithm showed significant opportunities to increase spectrum utilisation while keeping GoS at a particular level and ensuring a higher profit. We have also shown that our proposed optimisation solution not only reduce the total borrowing cost of the SNO but also finds maximum spectrum access under any allocated budget.

\section{REFERENCES}

[1] M. Matinmikko, H. Okkonen, M. Palola, S. Yrjola, P. Ahokangas, and M. Mustonen, "Spectrum sharing using licensed shared access: the concept and its workflow for lte-advanced networks," IEEE Wireless Communications, vol. 21, no. 2, pp. 72-79, 2014.

[2] A. Palaios, J. Riihijarvi, P. Mahonen, V. Atanasovski, L. Gavrilovska, P. Van Wesemael, A. Dejonghe, and P. Scheele, "Two days of spectrum use in europe," in 7th International ICST Conference on Cognitive Radio Oriented Wireless Networks and Communications (CROWNCOM), 2012, pp. 24-29.

[3] M. M. Buddhikot, P. Kolodzy, S. Miller, K. Ryan, and J. Evans, "Dimsumnet: new directions in wireless networking using coordinated dynamic spectrum," in Sixth IEEE International Symposium on a World of Wireless Mobile and Multimedia Networks. Ieee, 2005, pp. 78-85.

[4] M. M. Buddhikot and K. Ryan, "Spectrum management in coordinated dynamic spectrum access based cellular networks," in New Frontiers in Dynamic Spectrum Access Networks, 2005. DySPAN 2005. 2005 First IEEE International Symposium on. IEEE, 2005, pp. 299-307.

[5] M. M. Buddhikot, I. Kennedy, F. Mullany, and H. Viswanathan, "Ultrabroadband femtocells via opportunistic reuse of multi-operator and multi-service spectrum," Bell Labs Technical Journal, vol. 13, no. 4, pp. 129-143, 2009.

[6] OfCom, "Application of spectrum liberalisation and trading to the mobile sector - A further consultation," http://stakeholders.ofcom.org.uk/binaries/consultations/spectrumlib/anne xes/annex10.pdf, The Office of Communications, Tech. Rep., 2009.

[7] S. Li, J. Huang, and S.-Y. R. Li, "Dynamic profit maximization of cognitive mobile virtual network operator," IEEE Transactions on Mobile Computing, vol. 13, no. 3, pp. 526-540, 2014

[8] V. Sridhar and R. Prasad, "Towards a new policy framework for spectrum management in india," Telecommunications Policy, vol. 35, no. 2, pp. 172-184, 2011.

[9] A. Bourdena, E. Pallis, G. Kormentzas, and G. Mastorakis, "A prototype cognitive radio architecture for tvws exploitation under the real time secondary spectrum market policy," Physical Communication, vol. 10, pp. 159-168, 2014.

[10] J. Lunden, V. Koivunen, and H. V. Poor, "Spectrum exploration and exploitation for cognitive radio: Recent advances," IEEE Signal Processing Magazine, vol. 32, no. 3, pp. 123-140, 2015.

[11] F. Akhtar, M. H. Rehmani, and M. Reisslein, "White space: Definitional perspectives and their role in exploiting spectrum opportunities," Telecommunications Policy, vol. 40, no. 4, pp. 319-331, 2016.

[12] C. Jiang, Y. Chen, K. R. Liu, and Y. Ren, "Optimal pricing strategy for operators in cognitive femtocell networks," IEEE Transactions on Wireless Communications, vol. 13, no. 9, pp. 5288-5301, 2014.

[13] D. S. Palguna, D. J. Love, and I. Pollak, "Secondary spectrum auctions for markets with communication constraints," IEEE Transactions on Wireless Communications, vol. 15, no. 1, pp. 116-130, 2016.

[14] H. Zhang, C. Jiang, X. Mao, and H.-H. Chen, "Interference-limited resource optimization in cognitive femtocells with fairness and imperfect spectrum sensing," IEEE Transactions on Vehicular Technology, vol. 65 , no. 3, pp. 1761-1771, 2016.

[15] A. Afana, V. Asghari, A. Ghrayeb, and S. Affes, "On the performance of cooperative relaying spectrum-sharing systems with collaborative distributed beamforming," IEEE Transactions on Communications, vol. 62, no. 3, pp. 857-871, 2014.

[16] L. Wei, R. Q. Hu, Y. Qian, and G. Wu, "Energy efficiency and spectrum efficiency of multihop device-to-device communications underlaying cellular networks," IEEE Transactions on Vehicular Technology, vol. 65, no. 1 , pp. $367-380,2016$. 


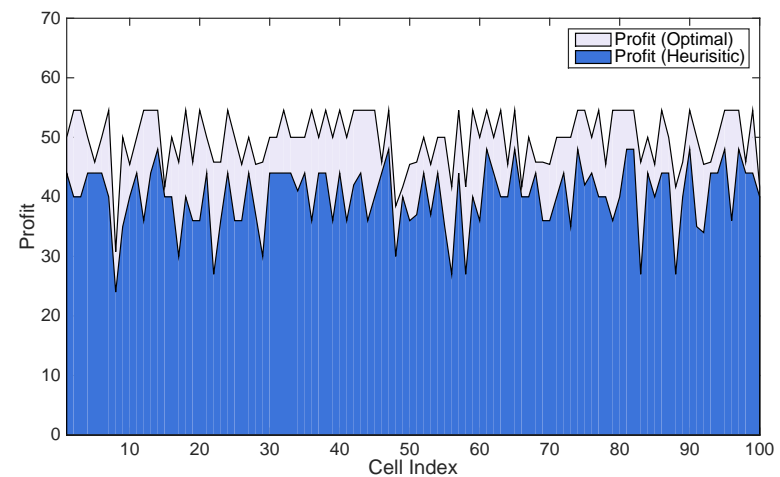

(a)

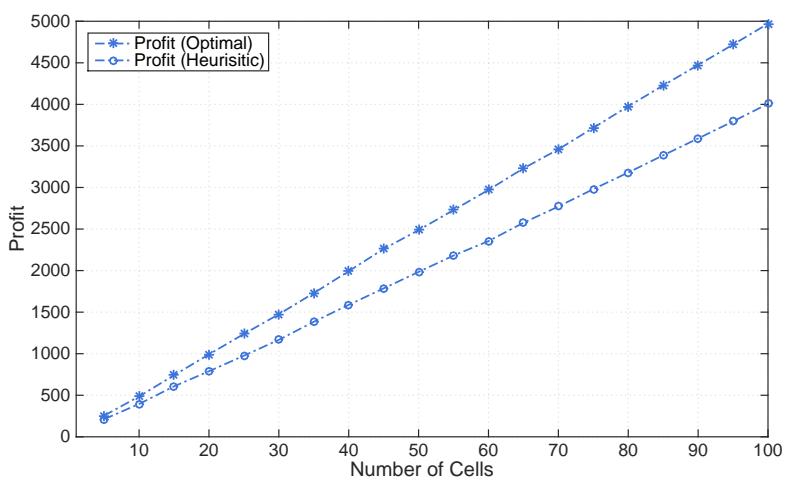

(b)

Fig. 2: Profit with random and optimal selection for (a) per cell and (b) total number of cells.

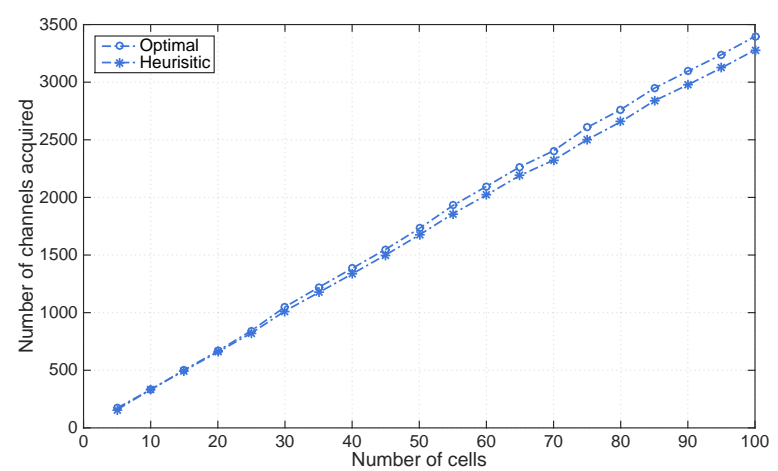

Fig. 3: Bandwidth acquisition of the SNO for spectrum borrowing by the optimal and heuristic algorithms.
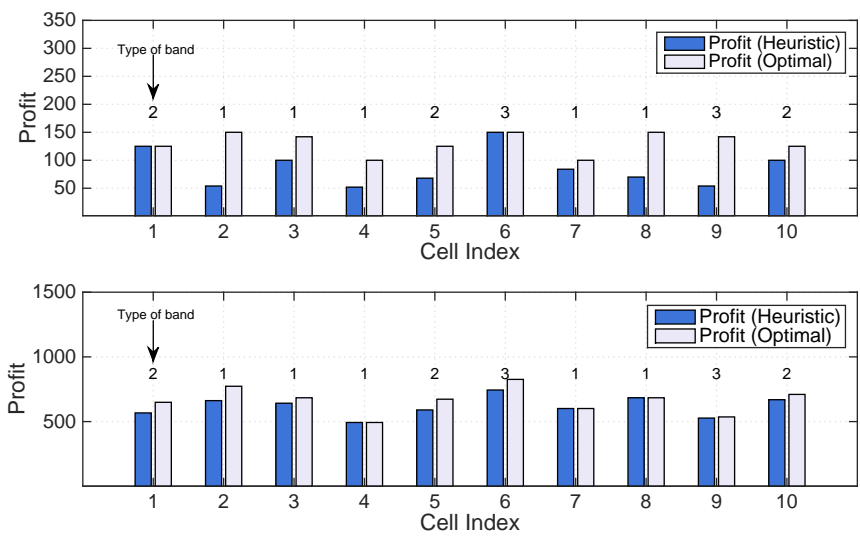

Fig. 4: Effect of spectrum borrowing on profit with budget $=50$ (top) and budget $=500$ (bottom).

[17] Y. Zhang, C. Lee, D. Niyato, and P. Wang, "Auction approaches for resource allocation in wireless systems: A survey," IEEE Communications Surveys \& Tutorials, vol. 15, no. 3, pp. 1020-1041, 2013.

[18] C. A. Gizelis and D. D. Vergados, "A survey of pricing schemes in wireless networks," IEEE Communications Surveys \& Tutorials, vol. 13, no. 1 , pp. 126-145, 2011
[19] X. Kang, R. Zhang, and M. Motani, "Price-based resource allocation for spectrum-sharing femtocell networks: A stackelberg game approach," IEEE Journal on Selected Areas in Communications, vol. 30, no. 3, pp. 538-549, 2012.

[20] A.-H. Mohsenian-Rad, V. W. Wong, and V. Leung, "Two-fold pricing to guarantee individual profits and maximum social welfare in multi-hop wireless access networks," IEEE Transactions on Wireless Communications, vol. 8, no. 8, pp. 4110-4121, 2009.

[21] N. Tran, L. B. Le, S. Ren, Z. Han, and C. S. Hong, "Joint pricing and load balancing for cognitive spectrum access: Non-cooperation versus cooperation," IEEE Journal on Selected Areas in Communications, vol. 33, no. 5, pp. 972-985, May 2015.

[22] R. Abozariba, M. Asaduzzaman, and M. Patwary, "Radio resource sharing framework for cooperative multi-operator networks with dynamic overflow modelling," IEEE Transactions on Vehicular Technology, 2016, In press.

[23] J. W. Mwangoka, P. Marques, and J. Rodriguez, "Broker based secondary spectrum trading," in Cognitive Radio Oriented Wireless Networks and Communications (CROWNCOM), 2011 Sixth International ICST Conference on. IEEE, 2011, pp. 186-190.

[24] S. Sengupta and M. Chatterjee, "An economic framework for dynamic spectrum access and service pricing," IEEE/ACM Transactions on Networking (TON), vol. 17, no. 4, pp. 1200-1213, 2009.

[25] M. Khaledi and A. A. Abouzeid, "Dynamic spectrum sharing auction with time-evolving channel qualities," IEEE Transactions on Wireless Communications, vol. 14, no. 11, pp. 5900-5912, 2015.

[26] Y. Wu, Q. Zhu, J. Huang, and D. H. Tsang, "Revenue sharing based resource allocation for dynamic spectrum access networks," IEEE Journal on Selected Areas in Communications, vol. 32, no. 11, pp. 2280-2296, 2014.

[27] I. Sugathapala, I. Kovacevic, B. Lorenzo, S. Glisic, and Y. M. Fang, "Quantifying benefits in a business portfolio for multi-operator spectrum sharing," IEEE Transactions on Wireless Communications, vol. 14 no. 12, pp. 6635-6649, 2015.

[28] Y. Song, C. Zhang, Y. Fang, and P. Lin, "Revenue maximization in time-varying multi-hop wireless networks: A dynamic pricing approach," IEEE Journal on Selected Areas in Communications, vol. 30, no. 7, pp. $1237-1245,2012$.

[29] L. Gao, J. Huang, Y.-J. Chen, and B. Shou, "An integrated contract and auction design for secondary spectrum trading," IEEE Journal on Selected Areas in Communications, vol. 31, no. 3, pp. 581-592, 2013.

[30] D. Niyato and E. Hossain, "Competitive pricing for spectrum sharing in cognitive radio networks: Dynamic game, inefficiency of nash equilibrium, and collusion," IEEE Journal on Selected Areas in Communications, vol. 26, no. 1, pp. 192-202, 2008. 